\title{
Protocol Test Sequence Generation Using MUIOS Based on TSP Problem
}

\author{
G. Prem Kumar and P. Venkataram \\ Department of Electrical Communication Engineering \\ Indian Institute of Science; Bangalore - 560 012, INDIA \\ (Tel: (+91) (080) 3340855; Fax: (+91) (080) 3347991 \\ e-mail : \{prem,pallapa\}@ece.iisc.ernet.in)
}

\begin{abstract}
In this paper, a method for optimal test sequence generation using Multiple Unique Input/Output Sequences (MUIOS) is presented. The test sequence generation problem using MUIOS is viewed as an asymmetric travelling sales person problem and is solved using simulated annealing methodology. This technique is applied to various test cases and the results are compared with the results obtained by using rural Chinese postman algorithm.
\end{abstract}

\section{Keywords}

Protocol conformance testing, formal methods for test sequence generation, multiple input/output sequences, travelling sales person problem, simulated annealing, rural Chinese postman algorithm.

\section{INTRODUCTION}

Conformance testing for communication protocols is to test whether a protocol under test, conforms to the specifications laid down during the design (Aho, 1991). The conformance testing of protocols is being done in two ways (Sarikaya, 1984) (Sarikaya, 1987) (Linn, 1989/1990). First approach is to build a test system, which simulates the protocol. These test systems designed by users and suppliers to their requirements, often have following disadvantages : (a) it is a costly effort to build individual testers, and (b) standardization of protocol products is difficult. Second approach deals with generation of efficient test cases for testing the implemented protocol product. The desirable features are short test sequences and less human intervention. Following the second way of conformance testing, this paper gives an efficient method of test sequence generation.

The formal methods used to generate conformance test sequences, that are modeled as Finite State Machines (FSMs) (Kohavi, 1978) are Transition Tours (T-method) (Bosik, 1991) (Naito, 1981) Distinguishing Sequence (D-method) (Gonenc, 1970) (Ural, 1992) (Chow, 1978), Characterizing Sequences (W-method) (Bosik, 1991) and Unique 
Input/Output Sequences (U-method) (Aho, 1991) (Bosik, 1991) (Sidhu, 1989) (Ural, 1992) (Sabnani, 1988) (Miller, 1993).

We formulate the problem of test sequence generation (TSG) based on MUIOS as an asymmetric travelling sales person problem (we call this as TSP) where sub-sequences (defined later) corresponding to an edge of the FSM are considered as a node and bridge length between the nodes as elements of the TSP-matrix. We solve the TSP problem thus formulated by using Simulated Annealing (SA) methodology.

In Section 2, the notation used in this paper and an overview of some of the protocol test sequence generation methods are introduced. In Section 3, the asymmetric travelling sales person problem and its solution using Simulated Annealing methodology are described. Section 4 explains the method of test sequence generation. Section 5 illustrates the proposed method with an example. Section 6 presents the results and comparison of the proposed method with the one solved using Rural Chinese Postman (RCP) problem. Finally, some concluding remarks are given in Section 7.

\section{NOTATION AND OVERVIEW OF FORMAL METHODS FOR TEST SEQUENCE GENERATION}

Before giving an overview of formal methods, we introduce the notation related to finite state machines, graph theory and protocol conformance testing, that is followed in this paper.

\subsection{Definitions}

Definition 1: A protocol represented as a FSM consists of a set of states and a set of transitions. It can be modelled as a directed graph $G=(V, E)$, where $V$ is a set of vertices corresponding to the states of the FSM and $E$ is a set of edges corresponding to the transitions of the FSM. $G$ is called as FSM graph.

Definition 2: A FSM $M$, is deterministic if there is at most one transition defined for each input at each state of $M$.

Definition 3: States $s_{i}$ and $s_{j}$ of $M$ are equivalent if they have identical edges.

Definition 4: $M$ is minimal if none of its states are equivalent.

Definition 5: $M$ is strongly connected if there exists a path between every pair of states.

Definition 6: $M$ is fully specified, if every state can accept all the possible inputs. Otherwise, $M$ is partially specified.

Definition 7: A state $s_{i}$ has a self loop if it has a transition that starts and ends in $s_{i}$.

Definition 8: A tour in a graph $G(V, E)$ is a sequence of consecutive edges which starts and ends at the same vertex in $V$.

Definition 9: A graph $G(V, E)$ is symmetric if for every vertex $v_{i} \in V$, the indegree of $v_{i}$ equals its outdegree.

Definition 10: An Euler tour in $G$ is a tour that contains every edge of $G$ exactly once. 
Definition 11: Chinese Postman Problem is defined as finding the minimum cost tour of a strongly connected directed graph $G$, that contains every edge of $G$ at least once.

Definition 12: Rural Chinese Postman ( $R C P$ ) problem is to find a tour in a strongly connected directed graph $G$ such that it traverses some subset of edges in $G$ at least once with minimum cost.

Definition 13: Controllability of a protocol is the ability to bring the implementation into a desired state.

Definition 14: Observability of a protocol is the ability to observe the current state of the protocol (this is generally done by looking at the output(s) generated).

In a protocol specification using FSM, testing an edge $\left(s_{i}, a_{p} / x_{q}, s_{j}\right)$ is done in three steps. In the first step, place the FSM in state $s_{i}$, second step is to apply the input $a_{p}$ and observe the output $x_{q}$ and the third step is to verify that the FSM entered into state $s_{j}$.

Definition 15: Reset edges are those edges that bring $M$ from any state of the FSM to the initial state.

We discuss, briefly, four formal methods for test sequence generation and their implementation as automated software tools.

\subsection{T-method}

Transition Tour (Bosik, 1991) (Naito, 1981) is an input sequence of FSM $M$, called TT, that starts at some state $s_{i}$, visits all the edges of $M$ at least once and comes back to $s_{i}$. One method of generating TT is to make $M$ (i.e., its graph $G$ ) symmetric (call that obtained graph as $G *$ ) such that it replicates minimum number of edges of $G$, which is solved as a linear programming problem, and finding an Euler tour of $G *$.

\section{$2.3 \quad$ D-method}

In Distinguishing Sequence method (Gonenc, 1970) (Chow, 1978), there is a sequence of inputs $I$, called $D S$, when applied to all the states of $M$, results in different outputs. One method of generating DS is to find the minimal covering of @(i,DS-diagram) (Kohavi, 1978) (where $i \in I$ and the symbol '@' denotes the concatenation operation on strings). One of the necessary conditions for a FSM to have DS is that, the FSM be fully specified.

\subsection{W-method}

Characterizing Sequence method (Bosik, 1991) is used only when $M$ does not have a DS. The idea here is to concatenate some more input transitions to form a partial $D S$, called $C S$, that distinguishes a state from a subset of those states which are responding with same output. The set of such input sequence of a FSM is called the characterizing set $W$ of the FSM. Once $W$ is obtained, the test sequence can be built using the method used in DS method. Like DS method, in this case also, the FSM needs to be fully specified. 


\subsection{U-method}

In this method, a Unique Input/Output (UIO) sequence is obtained for each state of the FSM. For example, if $\mathrm{UIO}_{j}$ is $(a / x, b / y)$ then no state other than $j$ will generate the output sequence $x, y$ for the input sequence $a, b$. To each edge $e_{i j}$, UIO $j$ is concatenated (together is called a sub-sequence, defined in detail later) and all such sub-sequences are joined in one of the several ways (Aho, 1991) (Bosik, 1991) (Miller, 1993) by using bridge sequences to build a test sequence. In (Sabnani, 1988), it is shown that U-method can be used to generate test sequences if combined with the rural Chinese postman algorithm, an NP-complete problem, for the graphs with two sufficient but not necessary conditions : FSM has reset feature and each state of the FSM has a self loop.

In a case, when some states have more than one UIO sequences (of minimal length), they are termed Multiple Unique Input/Output (MUIO) sequences. Then, for each state, choose one of the MUIO sequences, that gives the shortest test sequence. Many refinements of MUIOS are reported all of which attempt to minimize the length of the test sequence and yet have the properties of controllability and observability (Aho, 1991) (Lombardi, 1992) (Shen, 1992).

\section{THE TSP PROBLEM}

Travelling Sales Person problem is an example of a computationally hard problem (Rosenkrantz, 1977). In this problem, a sales person starts at an initial city and visits all $n$ cities exactly once so that the effective length of the tour is optimal (minimal). In asymmetric travelling sales person problem, where $d_{i j} \neq d_{j i}$, there are (n-1)! distinct tours with different lengths. Here, $d_{i j}$ represents the distance between the cities $i$ and $j$. Many solutions have been proposed to solve the TSP problem. Some of them are Nearest Neighbour approach, Branch and Bound algorithm, Linear Search, and Simulated Annealing. Since Simulated Annealing is used in this work, we briefly present about the first three methods and describe Simulated Annealing methodology in detail. In Nearest Neighbour approach, starting from a given city, the unvisited nearest neighboring city is visited next till the tour is complete (Rosenkrantz, 1977). In Branch and Bound algorithm (Gillet, 1979), an attempt is made to find a solution using Assignment Problem (AP). If AP fails to find a solution and results in sub-tours then Branch and Bound strategy is again applied to solve all the sub-problems using AP. The process is repeated till a tour with smallest bound is obtained. In Linear Search method (Kanellakis, 1980), the TSP problem is formulated as no-wait flowshop scheduling problem.

\subsection{Simulated Annealing}

Simulated Annealing concept is derived from annealing of metals in metallurgy. In annealing, metals are heated to high temperatures and are cooled down slowly and gradually. At each decrement in the value of temperature, the metal is allowed to reach the stable state, so that when it is cooled completely, it reaches thermal equilibrium. This concept is used to solve many optimization problems (Aarts, 1990) (Kirkpatrik, 1983). We use this technique to solve the test sequence generation problem by formulating it as a TSP problem. We discuss, here, the Simulated Annealing procedure (TSP-SA), to solve TSP problem. 


\subsubsection{Functioning of TSP-SA}

The procedure TSP-SA (see Figure 1) uses two basic functions : $O b j-f n()$ and Perturb(). $O b j-f n(R)$ returns length of the given tour $R$ of a graph with $n$ nodes. Initially tour $R$ is either selected randomly with a fixed initial node or taken from the user. $p$ is the number of current outer loop iterations. $\operatorname{Perturb}\left(R^{0}, p\right)$ perturbs the route vector $R^{0}$ at $p$ th iteration. Here two nodes, $i, j(1 \leq \mathrm{i}, \mathrm{j} \leq \mathrm{n}-1)$, of the route vector $R^{0}$ are randomly selected and swapped. ( $\mathrm{i}, \mathrm{j} \neq 0$ since the initial city is fixed.) $s$ is the cooling rate of the annealing process. random $(0,1)$ generates a value between 0 and 1 . The iterative values, $T_{i}$ and $T_{f}$ are analogous to the initial and final temperatures used in annealing of metals. $T_{i}$ and $T_{f}$ have to be selected properly based on the value, $\left(V_{0}-V_{1}\right)$ (Aarts, 1990). We select $T_{i}$ and $T_{f}$ values as 1000 and 10 respectively for $\left(V_{0}-V_{1}\right)$ of order 10. $s$ should be less than and very close to 1 . We set $\mathrm{s}=0.975$.

Procedure TSP-SA(input: $\mathrm{T}_{i}, \mathrm{~T}_{f}$, Max-ite; output: $\mathrm{R}(1 . . \mathrm{n})$ )

begin

var $\mathrm{p}, \mathrm{k}:$ int

$V_{0}, V_{1}:$ real

Initialize $\mathrm{R}$ to $\mathrm{R}^{0}$,

$V_{0}=\mathrm{Obj}-\mathrm{fn}\left(\mathrm{R}^{0}\right), \mathrm{p}=0$

while $\left(\mathrm{T}_{i}>\mathrm{T}_{f}\right)$

begin

$\mathrm{k}=0$

while $(\mathrm{k}<$ Max-ite $)$

begin

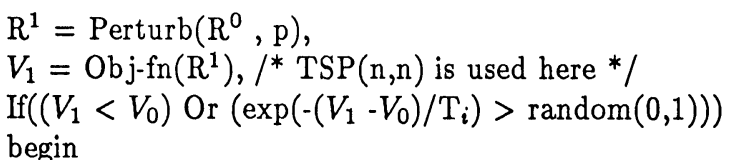

$\mathrm{R}^{0}=\mathrm{R}^{1}, V_{0}=V_{1}$, if $\left(\mathrm{Obj}-\mathrm{fn}\left(R^{0}\right)<\mathrm{Obj}-\mathrm{fn}(\mathrm{R})\right)$

begin

$\mathrm{R}=R^{0}$

end

end

$\mathrm{k}=\mathrm{k}+1$

end

end

$\mathrm{p}=\mathrm{p}+1, T_{i}=\mathrm{s} * T_{i}$

return $\mathrm{R}$

end

Figure 1 Procedure Simulated Annealing.

\section{TEST SEQUENCE GENERATION USING MUIOS, THE PRO- POSED METHOD}

In this section, for a protocol given in the form of a deterministic, strongly connected, minimal FSM, we formulate the conformance test sequence generation problem as an 
asymmetric travelling sales person problem and find a solution to this problem by using Simulated Annealing methodology as described in the previous section.

First, we introduce some terminology related to the proposed method and llustrate them using FSM $P 1$ (see Figure 2).

Definition $16:$ Sub-Sequence $\left(S S_{m k}\right)$ is a concatenation of an edge $e_{m} \in E$ of the FSM graph, $\mathrm{G}(\mathrm{V}, \mathrm{E})$, and $k$ th UIOS of its tail state. For example, the sub-sequences for the edge $e_{2}\left(s_{1}, \mathrm{~b} / \mathrm{y}, s_{3}\right)$ of FSM $P 1$ are,

$$
\begin{gathered}
S S_{21}:\left(s_{1}, \mathrm{~b} / \mathrm{y}, s_{3} ; s_{3}, \mathrm{a} / \mathrm{x}, s_{2} ; s_{2}, \mathrm{a} / \mathrm{x}, s_{1}\right), \\
S S_{22}:\left(s_{1}, \mathrm{~b} / \mathrm{y}, s_{3} ; s_{3}, \mathrm{~b} / \mathrm{y}, s_{1} ; s_{1}, \mathrm{a} / \mathrm{y}, s_{2}\right)
\end{gathered}
$$

since there are two UIOS corresponding to the tail state $s_{3}$, of $e_{2}$. Where as, edge $e_{1}\left(s_{1}, \mathrm{a} / \mathrm{y}, s_{2}\right)$ has only one sub-sequence,

$$
S S_{11}:\left(s_{1}, \mathrm{a} / \mathrm{y}, s_{2} ; s_{2}, \mathrm{a} / \mathrm{x}, s_{1} ; s_{1}, \mathrm{a} / \mathrm{y}, s_{2}\right) .
$$

Definition $17:$ Bridge length $\left(t_{i j}\right)$ is the distance between the tail state of sub-sequence $S S_{i k}$ and the head state of sub-sequence $S S_{j l}$. The $t_{i j}$ is an integer value which measures in terms of number of transitions essential to reach from tail state of $S S_{i k}$ to head state of $S S_{j l}$. The sequence joining them is called a bridge-sequence, $b_{i j}$. For example, bridge length $t_{21}$ between $S S_{22}$ and $S S_{11}$ is 1 and the corresponding bridge sequence $b_{21}$ is ' $a / x$ '. Similarly, the bridge length $t_{21}$ between the sub-sequence $S S_{21}$ and sub-sequence $S S_{11}$ is zero and the corresponding bridge-sequence $b_{21}$ is NULL.

Definition 18:TSG-graph is a directed graph $\operatorname{TSG}(N, B)$, where $N$ is a set of nodes, $n_{i} \in N, n_{i}=\left\{S S_{i k} \mid 1 \leq \mathrm{k} \leq \mathrm{U}, U\right.$ is the number of UIOS of the tail state of edge $e_{i}$ and $S S_{i k}$ are the sub-sequences corresponding to the edge $e_{i}$ of the FSM $\}$ and $B$ is a set of bridge-sequences, $b_{i j} \in B$ (the minimum bridge-sequence between the nodes $n_{i}$ and $n_{j}$ is represented as an edge). For example, the bridge-sequence $b_{21}$ entered as the edge of the TSG-graph is NULL and not ' $a / x$ ', since former is the minimum possible bridge-sequence between the nodes $n_{2}$ and $n_{1}$ of the TSG-graph.

Definition 19: A tour in TSG-graph always starts at the initial node, INODE (the sub-sequences in the INODE must have the initial state of the FSM as the head state) and traverses the minimal path across all the nodes. For example, in FSM P1 of Figure 2 , the INODE is $n_{1}$ or $n_{2}$, as both the nodes meet the requirement of having state $s_{1}$ as its head state.

Definition 20:TSG-matrix is a 2-D matrix, where each element, $T S G(i, j)$ contains two fields, first field representing the sub-sequence, $S S_{i k}$, chosen at node $n_{i}$ to traverse to $n_{j}$ with minimum bridge-sequence $b_{i j}$ and the second field representing the bridge-sequence $b_{i j}$. For example, the element, $T S G(2,1)$ contains $S S_{21} \mid$ NULL.

Definition 21: TSP-matrix is a 2-D matirx, where each element, $T S P(i, j)$ represents the bridge length, $t_{i j}$, between the nodes $n i$ and $n j$. For example, $T S P(2,1)$ represents the bridge length $t_{21}$, which is zero.

Definition 22: Overlap edges of two sub-sequences are those edges at the end of first sub-sequence, which overlap with same number of edges at the beginning of second subsequence. 


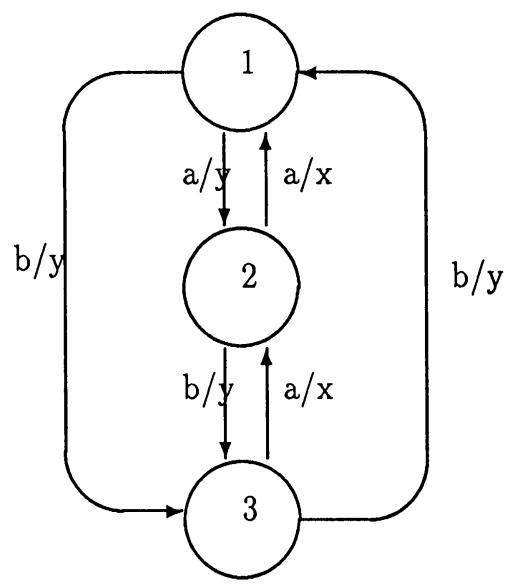

Figure 2 FSM P1.

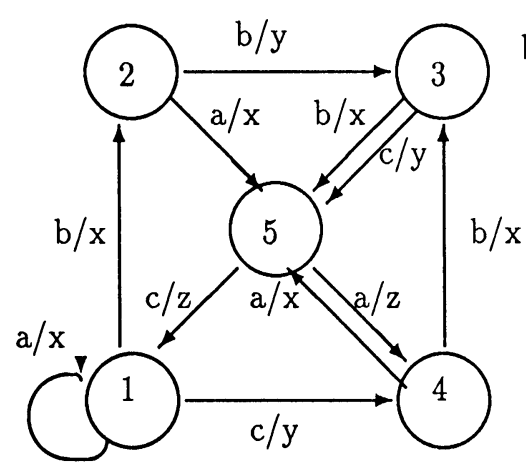

Figure 3 FSM P2.

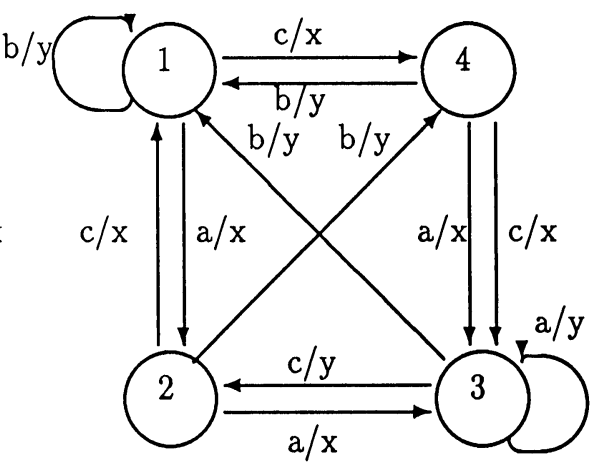

Figure 4 FSM P3.

Table 1 MUIOS for the FSMs shown in Figures 2-4

\begin{tabular}{|c|c|c|c|c|c|}
\hline \multicolumn{2}{|c|}{ FSM P1 } & \multicolumn{2}{|c|}{ FSM P2 } & \multicolumn{2}{|c|}{ FSM P3 } \\
\hline state & UIOS & state & UIOS & state & UIOS \\
\hline $\begin{array}{l}1 \\
2 \\
3\end{array}$ & $\begin{array}{l}a / y \\
a / x, a / y \\
* a / x, a / x \\
b / y, a / y\end{array}$ & $\begin{array}{l}2 \\
3 \\
4 \\
5\end{array}$ & $\begin{array}{c}\mathrm{a} / \mathrm{x}, \mathrm{a} / \mathrm{x} \\
\text { * b/x,a/x } \\
\mathrm{a} / \mathrm{x}, \mathrm{b} / \mathrm{x} \\
\mathrm{c} / \mathrm{y}, \mathrm{b} / \mathrm{x} \\
\mathrm{a} / \mathrm{x}, \mathrm{b} / \mathrm{y} \\
\mathrm{b} / \mathrm{y} \\
{ }^{*} \mathrm{~b} / \mathrm{x}, \mathrm{c} / \mathrm{z} \\
\mathrm{b} / \mathrm{x}, \mathrm{a} / \mathrm{z} \\
\mathrm{b} / \mathrm{x}, \mathrm{c} / \mathrm{y} \\
{ }^{*} \mathrm{c} / \mathrm{z} \\
\mathrm{a} / \mathrm{z}\end{array}$ & $\begin{array}{l}3 \\
4\end{array}$ & $\begin{array}{l}* a / x, c / x \\
a / x, a / x \\
* b / y, a / x, c / y \\
c / x, x / x, a / y \\
b / y, a / x, a / y \\
* c / y \\
\text { a } / y \\
* c / x, a / y \\
c / x, a / y\end{array}$ \\
\hline
\end{tabular}

* UIOS selected in case of SUIOS. 
Now, the solution to test sequence generation problem is to make a tour of the TSGgraph. Algorithm to generate protocol test sequences using the proposed method is given in Figure 5.

\section{Algorithm TSG-TSP}

begin

var $\mathrm{i}, \mathrm{j}, \mathrm{k}:$ int

1. Generate the MUIOS as given in (Sabnani, 1988).

2. Construct TSG-graph $T S G(N, B)$.

3. Select an $I N O D E$ in $\operatorname{TSG}(\mathrm{N}, \mathrm{B})$.

4. Construct $T S G(n, n)$ and $T S P(n, n)$ matrices, where $n$ is number of nodes in the TSG-graph.

5. Call $T S P-S A$ with $\operatorname{TSP}(\mathrm{n}, \mathrm{n})$ and obtain the route vector $R$.

6. Calculate the test sequence, TS, using the route vector, $R$ as follows : $\mathrm{k}=0, \mathrm{TS}=\mathrm{NULL}$

repeat

begin

$$
\begin{aligned}
& \mathrm{i}=\mathrm{R}(\mathrm{k}), \\
& \mathrm{j}=\mathrm{R}(\mathrm{k}+1) \text {, } \\
& \text { TS = @( TS, @(TSG(i,j).sub-sequence, TSG }(i, j) \text {.bridge-sequence })) \text {, } \\
& \mathrm{k}=\mathrm{k}+1
\end{aligned}
$$

end

until $(\mathrm{k} \geq(\mathrm{N}-1))$

7. Output TS.

end

Figure 5 Algorithm for test sequence generation using the proposed method.

\section{AN EXAMPLE}

We now illustrate the method of test sequence generation by the proposed TSG-TSP using FSM $P 1$ as an input protocol (see Figure 2) for the case of MUIOS without reset and without overlap.

In step 1 , we have the MUIOS of the FSM as shown in Table 1.

In step 2, construct the TSG-graph for the edges of the protocol, FSM P1,

$$
\mathrm{E}=\left\{s_{1}, \mathrm{a} / \mathrm{y}, s_{2} ; s_{1}, \mathrm{~b} / \mathrm{y}, s_{3} ; s_{2}, \mathrm{a} / \mathrm{x}, s_{1} ; s_{2}, \mathrm{~b} / \mathrm{y}, s_{3} ; s_{3}, \mathrm{~b} / \mathrm{y}, s_{1} ; s_{3}, \mathrm{a} / \mathrm{x}, s_{2}\right\} .
$$

The nodes of the TSG-graph are, $n_{i} \in N$, where $n_{i}$ represents a set of sub-sequences corresponding to $e_{i}$. They are :

$n_{1}$ :

$S S_{11}:\left(s_{1}, \mathrm{a} / \mathrm{y}, s_{2} ; s_{2}, \mathrm{a} / \mathrm{x}, s_{1} ; s_{1}, \mathrm{a} / \mathrm{y}, s_{2}\right)$

$n_{2}$ :

$S S_{21}:\left(s_{1}, \mathrm{~b} / \mathrm{y}, s_{3} ; s_{3}, \mathrm{a} / \mathrm{x}, s_{2} ; s_{2}, \mathrm{a} / \mathrm{x}, s_{1}\right)$,

$S S_{22}:\left(s_{1}, \mathrm{~b} / \mathrm{y}, s_{3} ; s_{3}, \mathrm{~b} / \mathrm{y}, s_{1} ; s_{1}, \mathrm{a} / \mathrm{y}, s_{2}\right)$ 
$n_{3}$ :

$S S_{31}:\left(s_{2}, \mathrm{a} / \mathrm{x}, s_{1} ; s_{1}, \mathrm{a} / \mathrm{y}, s_{2}\right)$

$n_{4}:$

$S S_{41}:\left(s_{2}, \mathrm{~b} / \mathrm{y}, s_{3} ; s_{3}, \mathrm{a} / \mathrm{x}, s_{2} ; s_{2}, \mathrm{a} / \mathrm{x}, s_{1}\right)$,

$S S_{42}:\left(s_{2}, \mathrm{~b} / \mathrm{y}, s_{3} ; s_{3}, \mathrm{~b} / \mathrm{y}, s_{1} ; s_{1}, \mathrm{a} / \mathrm{y}, s_{2}\right)$

$n_{5}$ :

$S S_{51}:\left(s_{3}, \mathrm{~b} / \mathrm{y}, s_{1} ; s_{1}, \mathrm{a} / \mathrm{y}, s_{2}\right)$

$n_{6}$ :

$S S_{61}:\left(s_{3}, \mathrm{a} / \mathrm{y}, s_{2} ; s_{2}, \mathrm{a} / \mathrm{x}, s_{1} ; s_{1}, \mathrm{a} / \mathrm{y}, s_{2}\right)$.

The edges of the TSG-graph are the minimum bridge-sequences between the nodes (given in TSG-matrix later). The TSG-graph for FSM P1 is shown in Figure 6.

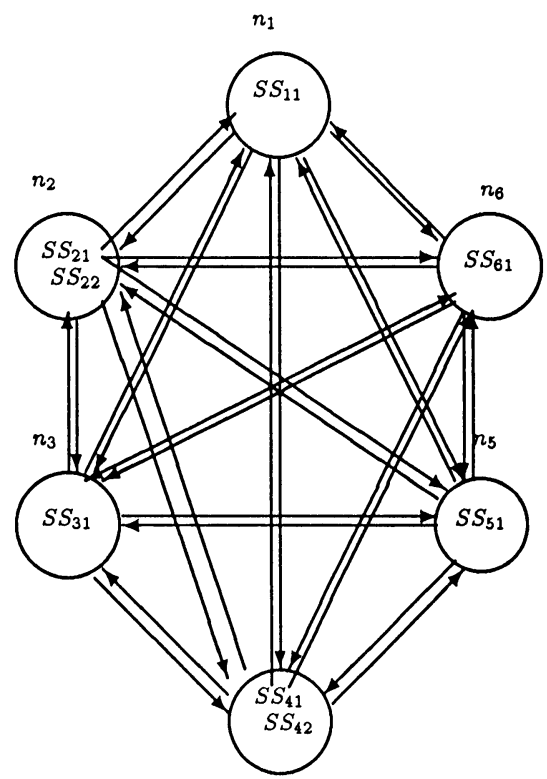

$n_{4}$

Figure 6 TSG-graph for the FSM P1 shown in Figure 2. 
In step 3 , select the INODE $n_{1}$ from the TSG-graph.

In step 4 , the TSG-matrix is :

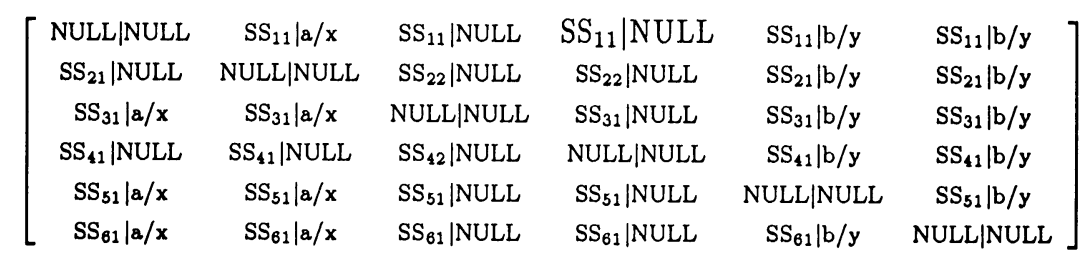

and the TSP-matrix is :

$$
\left[\begin{array}{cccccc}
999 & 1 & 0 & 0 & 1 & 1 \\
0 & 999 & 0 & 0 & 1 & 1 \\
1 & 1 & 999 & 0 & 1 & 1 \\
0 & 0 & 0 & 999 & 1 & 1 \\
1 & 1 & 0 & 0 & 999 & 1 \\
1 & 1 & 0 & 0 & 1 & 999
\end{array}\right]
$$

In step 5 , the procedure $T S P-S A$ is called with $\operatorname{TSP}(n, n)$, which returns the route vector $R=\left(\begin{array}{lllll}1 & 3 & 5 & 4 & 2\end{array}\right)$.

In step 6 , the test sequence is :

$$
a / y, a / x, a / y, a / x, a / y, b / y, b / y, a / y, b / y, a / x, a / x, b / y, a / x, a / x, b / y, a / x, a / x, a / y .
$$

The length of the test sequence is 18 .

\section{RESULTS}

We also present the results for other two FSMs shown in Figures 3 and 4. For each test case, we consider SUIOS without reset edges, with and without overlaps; MUIOS without overlaps, with and without reset edges. The results obtained by the proposed method are compared with results obtained using rural Chinese postman algorithm (see Table 2). 
Table 2 Comparison of length of the test sequences obtained by TSG-TSP and RCP

\begin{tabular}{|c|c|c|c|c|c|}
\hline \multirow{3}{*}{ Testcase } & \multicolumn{5}{|c|}{ Test Sequence Length } \\
\cline { 2 - 5 } & SUIO w/o reset edges & MUIO w/o overlap edges & $\begin{array}{c}\text { RCP with } \\
\text { reset and } \\
\text { w/.o overlap } \\
\text { using MUIOS }\end{array}$ \\
\hline P1 & 13 & 18 & 18 & 25 & 26 \\
P2 & 24 & 34 & 29 & 43 & 43 \\
P3 & 31 & 40 & 37 & 46 & 54 \\
\hline
\end{tabular}

\section{CONCLUSION}

In this paper, a technique for test sequence generation using MUIOS is described. The test sequence generation problem is considered as a TSP problem and is solved by using Simulated Annealing methodology. The results presented demonstrate that the method is at least as efficient as the method of test sequence generation using rural Chinese postman algorithm.

\section{ACKNOWLEDGMENT}

The authors would like to thank Prof. Sidhu and Dr. Motteler, University of Maryland, USA, for their helpful suggestions.

\section{REFERENCES}

Aarts E. and Korst J. (1990) Simulated Annealing and Boltzmann machines - A stochasic approach to combinatorial optimization and Neural computing, John Wiley \& Sons.

Aho A. V., Dahbura A. T., Lee D. and Uyar U. (1991), An optimization technique for protocol conformance test generation based on UIO subsequences and rural Chinese postman tours, IEEE trans. on Comm., 39(11), 1604-1615.

Bosik B. S. and Uyar M. U. (1991), Finite state machine based formal methods in protocol conformance testing : from theory to implementation, Comp. Networks and ISDN Systems, 22, 7-33.

Chow T. S. (1978) Testing software design modeled by finite-state machines, IEEE trans. on Software Engineering, SE-4(3), 178-187. 
Gillett B. E. (1979) Introduction to Operations Re search, A computer oriented algorithmic approach, Tata Mc Graw Hill Edition, New Delhi.

Gonenc G. (1970) A method for the design of fault detection experiments, IEEE trans. on Comp., 19, 551-558.

Kanellakis P. C. and Papadimitriou C. H. (1980) Linear search for the asymmetric travelling salesman problem, Operations Research, 28(5), 1086-1099.

Kirkpatrick S., Gelatt J. C. D. and Vecchi M. P. (1983) Optimization by Simulated Annealing, Science, 13 , 220(4598), 671-680.

Kohavi Z. (1978) Switching and Finite Automata Theory, Mc Graw Hill, New York.

Linn J. R. J. (1989/1990) Conformance testing for OSI protocols, Computer Networks and ISDN Systems, 18, 203-219.

Lombardi F. and Shen Y. N. (1992) Evaluation and improvement of fault coverage of conformance testing by UIO sequences, IEEE trans. on Comm., 40(8), 1288-1293.

Miller R. E. and Paul S. (1993) On the Generation of Minimal-Length Conformance Tests for Communication Protocols, IEEE/ACM trans. on Networking, 1(1), 116-129.

Naito S. and Tsunoyama M. (1981) Fault detection for sequential machines by transition-tours, in: Proc. of $11^{\text {th }}$ IEEE Fault Tolerant Computing Symp., 238-243.

Rosenkrantz D. J., Stearns R. E., and Lewis P. M. (1977) An analysis of several heursistics for the Travelling Salesman Problem, SIAM Jl. of Computing, 6, 563-581.

Sabnani K. and Dahbura A. T. (1988) A protocol testing procedure, Comp. Networks and ISDN Systems, 15, 285-297.

Sarikaya B. (1987) Conformance testing: Architectures and test sequences, Comp. Networks and ISDN Systems, 17, 111-126.

Sarikaya B. and Bochmann G. v. (1984) Synchronization and Specification issues in protocol testing, IEEE Software Engineering, Com-32(4), 389-395.

Shen Y. N., Lombardi F. and Dahbura A. T. (1992) Protocol conformance testing using Multiple UIO sequences, IEEE trans. on Comm., 40(8), 1282-1287.

Sidhu D. P. and Leung T. K. (1989) Formal methods for protocol testing : A detailed study, IEEE trans. of Software Engineering, 15(4), 413-426.

Ural H. (1992) Formal methods for test sequence generation, Comp. Comm., 15(5), $311-325$.

Prem Kumar Gadey received his B.Tech. ( Electronics \& Communication Engineering) from Sri Venkateswara University in 1990 and M.Tech. (Artificial Intelligence \& Robotics) from University of Hyderabad in 1992. Since then he is a Ph.D. student in department of Electrical Communicaiton Engineering, Indian Institute of Science, Bangalore. His major research interests include Communication Networks, Internetworking, Distributed Computing, Expert Systems and Artificial Neural Networks. Currently he is focussing on applying Artificial Intelligence techniques to the area of Network Management. He is a student member of IEEE Communication Society.

Pallapa Venkataram received his $\mathrm{Ph}$. D. degree from The University of Sheffield, England, in 1986. He is currently an Associate Professor in department of Electrical Communication Engineering, Indian Institute of Science, Bangalore, India. He worked in the areas of Distributed Databases, Communiation Protocols and AI applicaitons in Communication Networks and has published many papers in these areas. 